\title{
Direct Measurement of the Mass Difference Between Top and Antitop Quarks
}

\author{
T. Ferbel ${ }^{* \dagger}$ \\ Universities of Rochester and Maryland \\ E-mail: ferbel@pas.rochester.edu
}

\begin{abstract}
We present a measurement of the difference between the masses of top and antitop quarks in lepton+jets final states of top-antitop events in 1 event/fb of integrated luminosity collected by the D0 experiment at the Fermilab Tevatron Collider. The analysis is based on the "matrix element" method, with the purity of the signal enhanced by applying a neural-net-based $b$-tagging technique. This represents the first direct measurement of a mass difference between a quark and its antiquark and provides a test of CPT invariance in the quark sector. The measured value of $m_{t}-m_{\bar{t}}=3.8 \pm 3.7 \mathrm{GeV}$ is consistent with equality of the masses to within $\approx 2 \%$ of the mean mass of the top quark.
\end{abstract}

A copy of the poster presented at the conference appears on the next page. Also see the reports by T. Ferbel and C. Schwanenberger in these proceedings.

European Physical Society Europhysics Conference on High Energy Physics, EPS-HEP 2009,

July 16 - 222009

Krakow, Poland

\footnotetext{
* Speaker.

${ }^{\dagger}$ On behalf of the D0 collaboration, with thanks to Michael Wang for preparing the poster.
} 


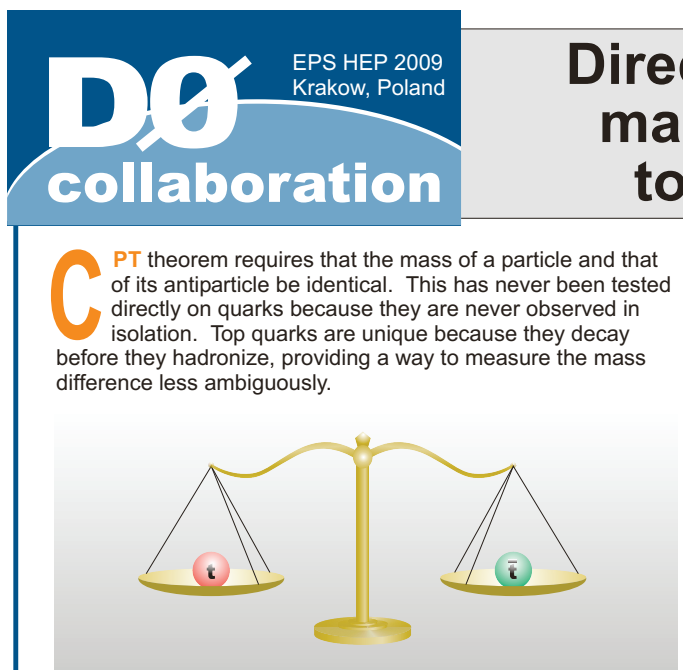

We search for any difference between the mass of the top and the antitop quark produced in $p \bar{p}$ collisions at $\sqrt{s}=1.96 \mathrm{TeV}$ using data corresponding to $1 \mathrm{fb}^{-1}$ of integrated luminosity collected with the D0 detector during Run II of the Fermilab Tevatron Collider. This analysis is based on $220 t \bar{t}$ candidate events in the lepton+jets channel selected by requiring one isolated lepton with high $p_{T}$, a large amount of missing transverse momentum, and exactly four high $p_{T}$ jets with at least one jet identified as a $b$ jet.

The analysis is conducted separately for the e+jets and $\mu+$ jets channels using the positively (negatively) charged leptons to tag the top (antitop) quark in each event. We do this analysis with the matrix element method developed at D0. For more details, please see arXiv:0906.1172 [hep-ex].

\section{Extracting the mass difference}

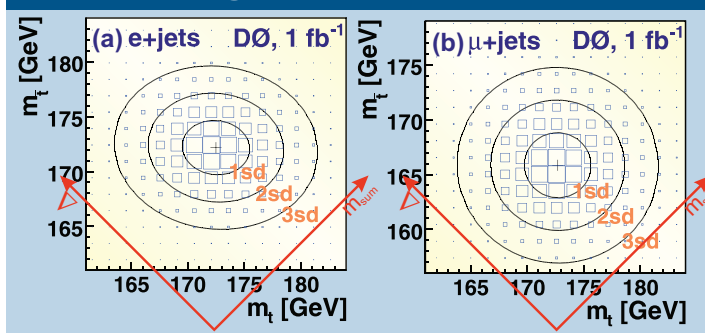

Figure 1: Fitted contours of equal probability for the $2 \mathrm{D}$ likelihoods as a function of $m_{t}$ and $m_{\bar{t}}$ for (a) e+jets and (b) $\mu+j$ ets data. The boxes, representing the bins in the 2D histograms of the likelihoods, display areas proportional to the bin contents, set equal to the value of the likelihood evaluated at the center of the bin.
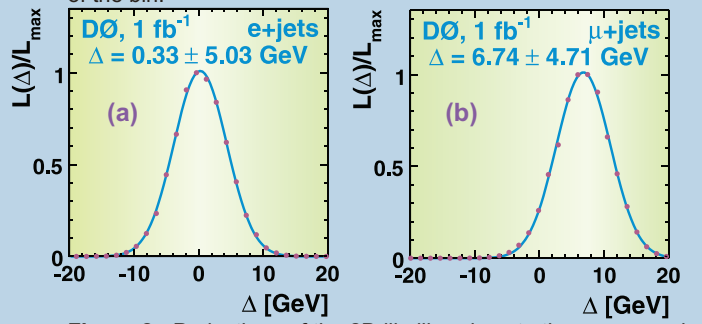

Figure 2: Projections of the 2D likelihoods onto the $\Delta=m_{t}-m_{i}$ axis (drawn in red in Fig. 1) for (a) e+jets and (b) $\mu+$ jets data. Values of $\Delta$ extracted from the projections are shown in each plot. Combining the extracted $\Delta$ from each channel gives $\Delta=3.8 \pm 3.4$ (stat.)

\section{Matrix element technique}

The matrix element (ME) method used in this analysis relies on the extraction of the properties of the top quark trhough a likelihood technique based on probability densities (PD) for each event, calculated from the ME for the two major processes ( $t \bar{t}$ and $W+$ jets) that contribute to the selected lepton+jets sample. The PD for each event is given in terms of the fraction of signal $(f)$ and of background $(1-f)$ in the data and the masses of the top quark $\left(m_{t}\right)$ and the antitop quark $\left(m_{i}\right)$ :

$$
P_{\text {evt }}=A(x)\left[f P_{\text {sig }}\left(x ; m_{t}, m_{\bar{t}}\right)+(1-f) P_{\mathrm{bkg}}(x)\right]
$$

where $x$ denotes the measured jet and lepton energies and angles, $A(x)$ is a function only of $x$ and accounts for the detector acceptance and efficiencies, and $P_{\text {sig }}$ and $P_{\text {bkg }}$ represent the PD for $t \bar{t}$ and $W+$ jets production, respectively.

$P_{\text {sig }}$ and $P_{\text {bkg }}$ are calculated by integrating over all possible parton states leading to the measured set $x$ through a convolution of the partonic differential cross section $d \sigma\left(y ; m_{t}, m_{\bar{t}}\right)$ with the PDs for the initial state partons and the transfer functions $W(y, x)$ that account for jet fragmentation effects and detector resolution

The parameters $m_{t}$ and $m_{i}$ are then determined from a likelihood constructed from the product of the $P_{\text {evt }}$ for all events.

\section{Result}

Table I: Summary of systematic uncertainties on $\Delta$.

\begin{tabular}{|c|c|}
\hline Source & $\begin{array}{l}\text { Uncertaint } \\
\quad(\mathrm{GeV})\end{array}$ \\
\hline \multicolumn{2}{|l|}{ Physics Modeling } \\
\hline Signal & \pm 0.85 \\
\hline PDF uncertainty & \pm 0.26 \\
\hline Background modeling & \pm 0.03 \\
\hline Heavy flavor scale factor & \pm 0.07 \\
\hline b-fragmentation & \pm 0.12 \\
\hline \multicolumn{2}{|l|}{ Detector Modeling } \\
\hline$b /$ light response ratio & \pm 0.04 \\
\hline Jet identification & \pm 0.16 \\
\hline Jet resolution & \pm 0.39 \\
\hline Trigger & \pm 0.09 \\
\hline Overall jet energy scale & \pm 0.08 \\
\hline Residual jet energy scale & \pm 0.07 \\
\hline Muon resolution & \pm 0.09 \\
\hline Wrong charge leptons & \pm 0.07 \\
\hline Asymmetry in $b \bar{b}$ response & \pm 0.42 \\
\hline \multicolumn{2}{|l|}{ Method } \\
\hline MC Calibration & \pm 0.25 \\
\hline$b$-tagging efficiency & \pm 0.25 \\
\hline Multijet contamination & \pm 0.40 \\
\hline Signal fraction & \pm 0.10 \\
\hline Total in quadrature & \pm 1.22 \\
\hline
\end{tabular}

We have measured the $t$ and $\bar{t}$ mass difference in $1 \mathrm{fb}^{-1}$ of data in lepton+jets $t \bar{t}$ event and find the mass difference to be:

$$
m_{t}-m_{\bar{t}}=3.8 \pm 3.7 \mathrm{GeV}
$$

corresponding to a relative mass difference of $(2.2 \pm 2.2) \%$. This is the first direct measurement of a mass difference between a quark and its antiquark partner. 